\title{
Health Care Costs of Adults Treated for Attention-Deficit/Hyperactivity Disorder Who Received Alternative Drug Therapies
}

\author{
Eric Q. Wu, PhD; Howard G. Birnbaum, PhD; Huabin F. Zhang, MD, MPH; \\ Jasmina I. Ivanova, MA; Elaine Yang, PhD; and David Mallet, MBA
}

\begin{abstract}
BACKGROUND: Many therapies exist for treating adult attention-deficit/hyperactivity disorder (ADHD), also referred to as attention-deficit disorder (ADD), but there is no research regarding cost differences associated with initiating alternative ADD/ADHD drug therapies in adults.

OBJECTIVE: To compare from the perspective of a large self-insured employer the risk-adjusted direct health care costs associated with 3 alternative drug therapies for ADD in newly treated patients: extended-release methylphenidate (osmotic release oral system-MPH), mixed amphetamine salts extended release (MAS-XR), or atomoxetine.

METHODS: We analyzed data from a US claims database of 5 million beneficiaries from 31 large self-insured employers (1999-2004). Analysis was restricted to adults aged 18 to 64 years with at least 1 diagnosis of ADD/ADHD (International Classification of Diseases, Ninth Revision, Clinical Modification [ICD-9-CM] codes 314.0x-attention deficit disorder; 314.00-attentiondeficit disorder without hyperactivity; or 314.01—attention-deficit disorder with hyperactivity) and at least 1 pharmacy claim for OROS-MPH, MAS-XR, or atomoxetine identified using National Drug Codes. In preliminary analysis, we calculated the duration of index ADHD drug therapy as time from index therapy initiation to a minimum 60-day gap. Because the median duration of index ADHD drug therapy was found to be approximately 90 days, the primary measures were total direct medical plus drug costs and medical-only costs computed over 6 months following therapy initiation. Adults were required to have continuous eligibility 6 months before and 6 months after their latest drug therapy initiation and no ADHD therapy during the previous 6 months. Cost was measured as the payment amount made by the health plan to the provider rather than billed charges, and it excluded patient copayments and deductibles. Medical costs included costs incurred for all-cause inpatient and outpatient/other services. Costs were adjusted for inflation to 2004 U.S. dollars using the consumer price index for medical care. $T$ tests were used for descriptive cost comparisons. Generalized linear models (GLMs) were used to compare costs of adults receiving alternative therapies, adjusting for demographic characteristics, substance abuse, depression, and the Charlson Comorbidity Index.
\end{abstract}

RESULTS: Of the 4,569 patients who received 1 of these 3 drug therapies for ADHD, 31.8\% received OROS-MPH for a median duration of 99 days of therapy, $34.0 \%$ received MAS-XR for a median 128 days, and $34.2 \%$ received atomoxetine for a median 86 days. In the 6-month follow-up period, the mean (standard deviation) total medical and drug costs were $\$ 2,008(\$ 3,231)$ for OROS-MPH, $\$ 2,169(\$ 4,828)$ for MAS-XR, and $\$ 2,540(\$ 4,269)$ for atomoxetine-treated adults. The GLM for patient characteristics suggested that 6-month, risk-adjusted mean medical costs, excluding drug costs, for adults treated with OROS-MPH were $\$ 142$ less $(10.4 \%, \$ 1,220$ vs. $\$ 1,362)$ compared with MAS-XR $(P=0.022)$ and $\$ 132$ less $(9.8 \%, \$ 1,220$ vs. $\$ 1,352)$ compared with atomoxetine $(P=0.033)$; risk-adjusted mean medical costs were not significantly different between MAS-XR and atomoxetine. The GLM comparison of risk-adjusted total direct costs, including drug cost, was on average $\$ 156$ less $(8.0 \%, \$ 1,782$ vs. $\$ 1,938)$ for OROS-MPH compared with MAS-XR $(P=0.017)$ and $\$ 226$ less $(11.3 \%, \$ 1,782$ vs. $\$ 2,008)$ compared with atomoxetine $(P<0.001)$; the risk-adjusted total direct costs were not significantly different between MAS-XR and atomoxetine. Two high-cost outliers

Note: An editorial on the subject of this article appears on pages 617-19 of this issue. (greater than 99.96th percentile, 1 each for OROS-MPH and atomoxetine) accounted for $\$ 47(30 \%)$ of the $\$ 156$ cost difference between OROS-MPH and MAS-XR and $\$ 11(5 \%)$ of the $\$ 226$ cost difference between OROS-MPH and atomoxetine, and the medical diagnoses for the highest-cost claims for these 2 outlier patients were unrelated to ADHD.

CONCLUSIONS: After adjusting for patient characteristics including substance abuse, depression, and the Charlson Comorbidity Index, adults treated with OROS-MPH had, on average, slightly lower medical and total medical and drug costs than those treated with MAS-XR or atomoxetine over the 6-month period after drug therapy initiation. Approximately $30 \%$ of the cost difference compared with MAS-XR was attributable to 1 high-cost outlier with medical diagnoses for the highest-cost claim that were unrelated to ADHD.

KEYWORDS: Attention-deficit/hyperactivity disorder, ADHD, Costs, Extendedrelease methylphenidate, OROS-MPH, Mixed amphetamine salts, MAS-XR, Atomoxetine

J Manag Care Pharm. 2007;13(7):561-69

Copyright@ 2007, Academy of Managed Care Pharmacy. All rights reserved.

What is already known about this subject

- Previous studies have indicated that MPH is a cost-effective treatment for children with ADHD

- Health care costs of alternative drug therapies in adults with ADHD have not been evaluated. Studies in adults have focused on the economic burden of ADHD associated with an increase in comorbidity rates, risk of accidents, and health care costs but have not provided information about the health care costs associated with initiating alternative ADHD treatments in adults with ADHD

\section{What this study adds}

- Over the 6-month period after drug therapy initiation, adults with ADHD initiated on OROS-MPH had, on average, $8 \%$ lower risk-adjusted total medical and pharmaceutical costs than did those initiated on MAS-XR and 11\% lower costs compared with atomoxetine. However, these differences were affected by 1 outlier case for each comparison with diagnoses, such as chronic kidney disease and acute myocardial infarction, that were unrelated to ADHD or to accidents.

A ttention-deficit/hyperactivity disorder (ADHD) is estimated to affect $4.4 \%$ of the working adult population in the United States. ${ }^{1}$ Historically, ADHD has been consid- 
ered a childhood condition; only recently has there been a heightened awareness among clinicians and researchers regarding ADHD in adulthood. ${ }^{2,3}$ Childhood ADHD persists into adulthood in up to $60 \%$ of diagnosed cases. ${ }^{4,5}$

The clinical features of ADHD-associated symptoms include poor concentration, general disorganization, tendency to leave projects incomplete, inattention, poor school/work performance, problems with time management, difficulty controlling temper, impulsivity, and being hyperfocused..$^{4-7}$ ADHD patients also have an increased prevalence of comorbid conditions such as asthma, anxiety, bipolar disorder, depression, drug or alcohol abuse, antisocial disorder, or oppositional disorder. ${ }^{2,8,9}$

The potential societal costs of adult ADHD are considerable. ADHD has been associated with an increased risk of accidents, which would have consequences regarding the use and cost of health care services. ${ }^{10}$ Productivity loss of adults with ADHD is estimated to be 35 days a year. ${ }^{1}$ The estimated burden of ADHD in the United States was \$31.6 billion in 2000 , and $45 \%$ of that burden was attributable to excess health care costs of family members of patients with ADHD. ${ }^{11}$ Compared with a matched cohort, ADHD patients had almost 3 times greater annual health care costs, and family members had approximately 1.9 times higher annual health care expenditures compared with a matched cohort of family members of non-ADHD patients. ${ }^{12}$

ADHD in adults is amenable to treatment and is best controlled by a combination of medications and psychosocial interventions. ${ }^{6,13,14}$ Common medications used for the treatment of ADHD in adults are stimulants (e.g., methylphenidate [MPH], mixed amphetamine salts [MAS], dextroamphetamine) or nonstimulants (e.g., atomoxetine), ${ }^{4,8,15-17}$ In the past, treatment options usually included either short- or intermediate-acting stimulants and antidepressants. ${ }^{18}$ Recently, stimulant products have entered the marketplace in extended-release formulations; the first methylphenidate product that lasts 12 hours with once-daily dosing is osmotic release oral system (OROS)-MPH, and the once-daily amphetamine preparation is MAS-XR. Atomoxetine hydrochloride is the first nonstimulant to receive an indication for ADHD in adults from the U.S. Food and Drug Administration. ${ }^{15}$ Both stimulant and nonstimulant treatments have been shown to be effective in improving ADHD symptoms. ${ }^{17,19}$ Stimulant therapy led to improvement in $65 \%$ to $75 \%$ versus $5 \%$ to $30 \%$ of patients randomized to placebo. ${ }^{19}$ Nonstimulant therapy has also been shown to improve symptoms assessed with the ADHD rating scales. ${ }^{17}$

Quantifying costs related to ADHD and to alternative therapies is important in order to understand the economic impact of the condition and provide a basis for the development of programs and policies to assist patients suffering from this disability. The objective of this article is to compare riskadjusted medical and total health care costs of adults diagnosed with ADHD initiated on extended-release methylphenidate
(OROS-MPH, Concerta), mixed amphetamine salts extended release (MAS-XR, Adderall XR), or atomoxetine (Strattera) from the perspective of a large self-insured employer.

\section{Methods}

\section{Data}

Our study is based on data from a deidentified administrative claims database maintained by Ingenix, Inc. (Eden Prairie, MN) containing medical and demographic information on privately insured employees, retirees, and their spouses and dependents from 31 large self-insured companies in the United States. The claims cover services provided from January 1999 through December 2004. The 31 companies have national operations, span a broad array of industries and occupations, and cover approximately 5 million beneficiaries. On average, each employer has 2 to 3 health plans. The database includes medical and pharmacy claims for all employees plus their spouses and dependents. Data on the monthly eligibility of beneficiaries are available, as well as employee demographic information such as age, gender, geographic region of residence, and employee status of the primary beneficiary. The formulary status of ADHD drug therapies for these health plans is not known.

\section{Sample Selection}

The study sample was drawn from all 31 companies and included adults between the ages of 18 and 64 years during the study period. Patients were included in the ADHD sample if they met the following criteria (outlined in the Figure):

- Adults aged 18 to 64 years with at least 1 ADHD diagnosis, defined as an International Classification of Diseases, Ninth Revision, Clinical Modification (ICD-9-CM) code of 314.0X (attention deficit disorder), 314.00 (attention deficit disorder without hyper-activity), or 314.01 (attention deficit disorder with hyper-activity) between January 1999 and December 2004.

- At least 1 pharmacy claim for OROS-MPH (National Drug Codes [NDC] 17314585002, 17314585102, 17314585202, 17314585302, 54868448900, 54868475900, $54868475901, \quad 54868475902, \quad 54868475903), \quad$ MAS-XR (NDC codes 54092038101, 54092038301, 54092038501, 54092038701,54092038901,54092039101,54868476001), or atomoxetine (NDC codes 00002322730, 00002322830, 00002322930, 00002323830, 00002323830).

- Continuous enrollment in a health care plan during the 6 months prior to and 6 months following the therapy index date. Therapy index date was the date of the most recent prescription fill date during the 5-year period from 1999 through 2004 for OROS-MPH, MAS-XR, or atomoxetine, with continuous eligibility 6 months prior to and after the claim date and no ADHD diagnosis or therapy during the previous 6 months (washout period). In addition to 


\section{FIGURE Sample Selection (Population of Approximately 5 Million Beneficiaries)}

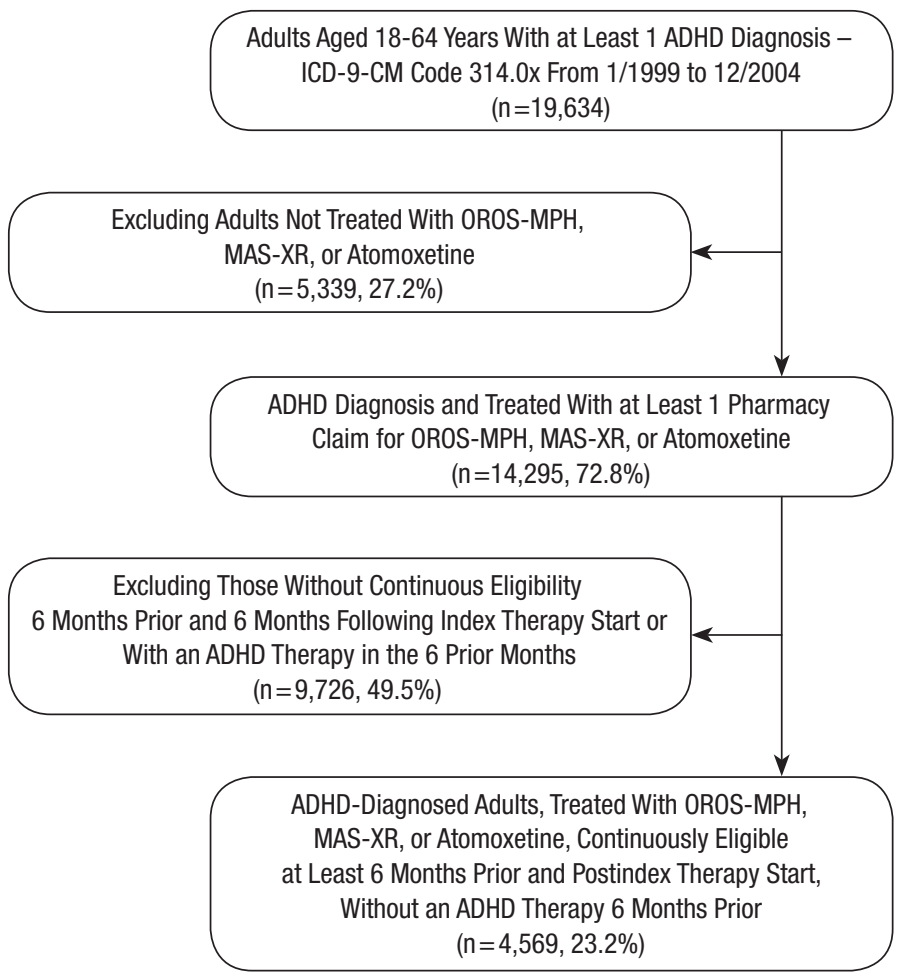

$A D H D=$ attention-deficit/hyperactivity disorder; ICD-9-CM = International Classification of Diseases, Ninth Revision, Clinical Modification; MAS-XR=mixed amphetamine salts extended release; OROS-MPH=osmotic release oral system extended-release methylphenidate.

forbidding the use of the comparator therapies OROS-MPH, MAS-XR, or atomoxetine, the washout period required no use of short- and intermediate- acting stimulant ADHD therapies.

\section{Cost Estimation}

Cost analyses were conducted from the third-party payer's perspective (i.e., costs were defined as payments to providers by third-party payers after subtraction of all member cost share including deductibles, coinsurance, and copayments). All costs were measured on a 6-month basis and adjusted for inflation to 2004 U.S. dollars using the consumer price index for medical care. The decision to estimate 6-month costs was based on preliminary analysis of the duration of therapy. Duration of therapy was defined as the time from index therapy initiation to a minimum 60-day gap in the index therapy supply. In the preliminary analysis, the overall median duration of index drug therapy was approximately 90 days.
Medical costs were calculated based on reimbursements from the employer to health care providers for inpatient care, hospital outpatient care (e.g., outpatient surgery), physician services, and emergency room visits, as well as other ancillary services (e.g., physical therapy, laboratory services). Costs were categorized in 2 mutually exclusive categories, inpatient services and outpatient/other services, based on the place of service code associated with each claim. We relied on place of service categories because revenue codes were not available in the data. Costs of inpatient services were defined using claims with a place of service specified as hospital inpatient, rehabilitation center, residential treatment center, or psychiatric facility. All other medical costs were grouped into an "outpatient and other costs" category that also included services with place of service specified as emergency treatment centers or hospital emergency rooms. Number of outpatient/other visits was defined as the summed number of unique days with a claim with place of service other than hospital inpatient, rehabilitation center, residential treatment center, or psychiatric facility. Total health care costs were defined as medical (inpatient and outpatient) plus prescription drug costs.

Costs attributable to psychotherapy, a subset of medical costs, were also reported. Psychotherapy encounters could have occurred in either an inpatient or outpatient setting, and we did not attempt to divide them into inpatient and outpatient subcategories. Psychotherapy costs were estimated using medical claims with Current Procedural Terminology (CPT) codes for psychotherapy (CPT codes 90804-90857, 96150-96155). Psychotherapy visits were defined as the summed number of unique days with at least 1 psychotherapy claim.

For each claim with an inpatient place of service, we identified the reason for the hospital use based on up to 2 primary or secondary diagnosis codes. The most frequently recorded diagnoses were designated as the reason for hospital services, irrespective of whether they appeared as primary or secondary on the claim.

About $0.1 \%$ of the medical claims had a missing amount paid. A procedure described as stratified hot-deck imputations, outlined by Little and Rubin, was applied to impute the missing paid amounts from randomly selected claims with complete paid amounts that contained the same procedure code, place of service, and type of service. ${ }^{20}$

\section{Analyses}

All analyses were performed on an intent-to-treat basis (i.e., adults were grouped by their index therapy). Statistical significance was evaluated at the 0.05 significance level. The proportions of patients with at least 1 inpatient place of service claim, emergency room claim (defined as a claim with a place of service in an emergency treatment center or hospital emergency room), and outpatient or other claim over the 6-month follow-up period were compared between OROS-MPH and MAS-XR and between 
Health Care Costs of Adults Treated for

Attention-Deficit/Hyperactivity Disorder Who Received Alternative Drug Therapies

TABLE 1 Baseline Characteristics of Adults Diagnosed With ADHD Receiving Different Drug Therapies

\begin{tabular}{|c|c|c|c|c|c|c|c|c|c|}
\hline \multirow{3}{*}{$\begin{array}{l}\text { Baseline } \\
\text { Characteristics } \\
\text { Age, mean [SD] }\end{array}$} & \multirow{2}{*}{\multicolumn{2}{|c|}{$\begin{array}{c}\text { OROS-MPH }(\mathrm{N}=1,452) \\
{[\mathrm{A}]}\end{array}$}} & \multirow{2}{*}{\multicolumn{2}{|c|}{$\frac{\text { MAS-XR }(\mathrm{N}=1,554)}{[\mathrm{B}]}$}} & \multirow{2}{*}{\multicolumn{2}{|c|}{$\begin{array}{c}\text { Atomoxetine }(\mathrm{N}=1,563) \\
{[\mathrm{C}]}\end{array}$}} & \multirow{3}{*}{$\begin{array}{c}P \text { Value } \\
{[\text { A] vs. [B] }} \\
0.009\end{array}$} & \multirow{3}{*}{$\begin{array}{c}P \text { Value } \\
{[\text { A] vs. }[\mathrm{C}]} \\
<0.001\end{array}$} & \multirow{3}{*}{$\begin{array}{c}P \text { Value } \\
{[B] \text { vs. }[\mathrm{C}]} \\
<0.001\end{array}$} \\
\hline & & & & & & & & & \\
\hline & 31.7 & [13.2] & 30.5 & {$[12.5]$} & 34.9 & [13.1] & & & \\
\hline Female, n (\%) & 582 & $(40.1 \%)$ & 704 & $(45.3 \%)$ & 664 & $(42.5 \%)$ & 0.004 & 0.181 & 0.113 \\
\hline Substance abuse, n (\%) & 29 & $(2.0 \%)$ & 53 & $(3.4 \%)$ & 51 & $(3.3 \%)$ & 0.018 & 0.031 & 0.819 \\
\hline Depression/anxiety, n (\%) & 357 & $(24.6 \%)$ & 423 & $(27.2 \%)$ & 416 & $(26.6 \%)$ & 0.100 & 0.202 & 0.704 \\
\hline $\begin{array}{l}\text { Charlson Comorbidity } \\
\text { Index, mean [SD] }\end{array}$ & 0.14 & {$[0.52]$} & 0.09 & {$[0.44]$} & 0.14 & {$[0.55]$} & 0.014 & 0.745 & 0.005 \\
\hline $\begin{array}{l}\text { Patients with a } \\
\text { hospitalization, n (\%) }\end{array}$ & 67 & $(4.6 \%)$ & 63 & $(4.1 \%)$ & 83 & $(5.3 \%)$ & 0.451 & 0.380 & 0.097 \\
\hline
\end{tabular}

* T tests were used to test for equality of means, and chi-squared tests were used to test for equality of proportions.

$A D H D=$ attention-deficit/hyperactivity disorder; MAS-XR = mixed amphetamine salts extended release; OROS-MPH = extended-release methylphenidate.

OROS-MPH and atomoxetine using chi-square tests.

Six-month medical (inpatient and outpatient) and drug costs were calculated for adults treated with OROS-MPH, MAS-XR, or atomoxetine. $T$ tests were used for a descriptive comparison of observed costs among the treatment groups. Multivariate regression models were used to compare medical and direct health care costs between OROS-MPH and each of the other 2 therapies while adjusting for baseline patient characteristics.

A generalized linear model (GLM) specification with a log link function and gamma distribution for the error term was used to resolve the issue of a skewed cost distribution common in claims data analysis. ${ }^{21}$ In contrast with the traditional logordinary least squares regression, GLM provides more robust coefficient estimates. ${ }^{22}$ Also, the log link function of the mean response enables coefficients to be directly back-transformed into the original dollar scale and avoids the issue of potentially biased estimates that may result from using the Duan smearing estimation method. ${ }^{23}$

Patient characteristics included age, gender, region, selected comorbidities (substance abuse and depression/anxiety), and the Charlson Comorbidity Index. Primary and secondary diagnoses during the 6 months prior to initiation of index therapy were used to identify the selected comorbidities and construct the Charlson Comorbidity Index. Substance abuse was defined as a diagnosis with 1 of the following ICD-9-CM codes: V65.42, 305.xx, 304.xx, 292.xx, 303.xx, 305.0x, or 291.xx. Depression/anxiety was defined as a diagnosis with ICD-9-CM codes 296.xx, V79.0x, 311.xx, or 300.0x. We chose to adjust for depression/anxiety and substance abuse based on prior studies reporting that patients diagnosed with ADHD were more likely to have anxiety, bipolar disorder, depression, drug or alcohol abuse, or conduct disorders compared with matched controls. ${ }^{9}$
Depression was found to be the most common comorbid condition among patients with ADHD. ${ }^{24}$ Another study reported that patients were more likely to receive atomoxetine compared with stimulants if they had a prior history of bipolar disorder, anxiety, substance abuse, or antidepressant use. ${ }^{25}$

The Charlson Comorbidity Index is a weighted sum of 17 comorbidities in which comorbidity weights are based on adjusted risk of 1-year mortality; the higher the Charlson Comorbidity Index, the higher the illness burden. ${ }^{26}$ ICD-9-CM diagnosis codes defined by Romano et al. were used to identify the included comorbidities: myocardial infarction, congestive heart failure, peripheral vascular disease, cerebrovascular disease, dementia, chronic pulmonary disease, rheumatologic disease, peptic ulcer disease, mild liver disease, moderate to severe liver disease, mild to moderate diabetes, diabetes with complications, hemiplegia or paraplegia, renal disease, any malignancy including lymphoma or leukemia, metastatic solid tumor, and AIDS. ${ }^{27}$ All analyses were performed using SAS Version 9.1 (SAS Institute, Cary, NC).

\section{Results}

\section{Demographics: 6-Month Preperiod}

Adults with a diagnosis of attention-deficit disorder (ADD)/ ADHD receiving OROS-MPH, MAS-XR, or atomoxetine were, on average, 32 years old. Approximately $43 \%$ of the adults in the study sample were female, $3 \%$ had a diagnosis related to substance abuse in the previous 6 months, and $26 \%$ had a depression/anxiety-related diagnosis in the previous 6 months (Table 1).

A descriptive comparison of demographic characteristics indicated that patients initiated with MAS-XR were the youngest and atomoxetine users were the oldest. OROS-MPH patients had a lower rate of substance abuse $(2.0 \%, P=0.018)$ than 
Health Care Costs of Adults Treated for

Attention-Deficit/Hyperactivity Disorder Who Received Alternative Drug Therapies

TABLE $2 \longdiv { 6 - m o n t h ~ U t i l i z a t i o n ~ a n d ~ C o s t s ~ f o r ~ A d u l t s ~ D i a g n o s e d ~ W i t h ~ A D H D ~ R e c e i v i n g ~ D i f f e r e n t ~ T h e r a p i e s ~ }$

\begin{tabular}{|c|c|c|c|c|c|c|c|c|c|}
\hline \multirow{4}{*}{$\begin{array}{l}\text { Mean [SD] } \\
\text { Median } \\
\begin{array}{l}\text { Duration of index ADHD } \\
\text { therapy (in days) } \dagger\end{array}\end{array}$} & \multirow{2}{*}{\multicolumn{2}{|c|}{$\begin{array}{c}\begin{array}{c}\text { OROS-MPH } \\
(\mathrm{N}=1,452)\end{array} \\
{[\mathrm{A}]}\end{array}$}} & \multirow{2}{*}{\multicolumn{2}{|c|}{$\begin{array}{c}\text { MAS-XR } \\
(\mathrm{N}=1,554) \\
{[\mathrm{B}]} \\
\end{array}$}} & \multirow{2}{*}{\multicolumn{2}{|c|}{$\begin{array}{c}\text { Atomoxetine } \\
(\mathrm{N}=1,563) \\
{[\mathrm{C}]}\end{array}$}} & \multirow{3}{*}{$\begin{array}{c}\frac{P \text { Value }^{*}}{\text { [A] vs. [B] }} \\
0.001\end{array}$} & \multirow{3}{*}{$\begin{array}{c}\text { P Value* } \\
\text { [A] vs. [C] } \\
<0.001\end{array}$} & \multirow{3}{*}{$\begin{array}{c}\text { P Value }^{*} \\
{[\mathrm{~B}] \text { vs. }[\mathrm{C}]} \\
<0.001\end{array}$} \\
\hline & & & & & & & & & \\
\hline & 107 & [66] & 115 & {$[65]$} & 96 & [65] & & & \\
\hline & 99 & & 128 & & 86 & & & & \\
\hline $\begin{array}{l}\text { No. patients (\%) on index } \\
\text { ADHD drug therapy at } 180 \text { days }\end{array}$ & 546 & $(37.6 \%)$ & 655 & $(42.2 \%)$ & 443 & $(28.3 \%)$ & 0.011 & $<0.001$ & $<0.001$ \\
\hline $\begin{array}{l}\text { Minimum/maximum duration } \\
\text { of ADHD index therapy }\end{array}$ & $3 / 180$ & & $7 / 180$ & & $4 / 180$ & & & & \\
\hline ADHD drug cost & $\$ 282$ & {$[\$ 215]$} & $\$ 322$ & {$[\$ 250]$} & $\$ 392$ & [\$298] & $<0.001$ & $<0.001$ & $<0.001$ \\
\hline & $\$ 246$ & & $\$ 275$ & & $\$ 325$ & & & & \\
\hline Total drug cost & $\$ 757$ & {$[\$ 1,058]$} & $\$ 748$ & {$[\$ 1,163]$} & $\$ 959$ & {$[\$ 1,481]$} & 0.812 & $<0.001$ & $<0.001$ \\
\hline & $\$ 449$ & & $\$ 490$ & & $\$ 626$ & & & & \\
\hline Inpatient services $\ddagger, n(\%)$ & 50 & $(3.4 \%)$ & 75 & $(4.8 \%)$ & 80 & $(5.1 \%)$ & 0.058 & 0.024 & 0.708 \\
\hline Hospital inpatient cost $\S$ & $\$ 139$ & {$[\$ 1,336]$} & $\$ 261$ & {$[\$ 2,060]$} & $\$ 334$ & {$[\$ 2,480]$} & 0.053 & 0.007 & 0.372 \\
\hline & $\$ 0$ & & $\$ 0$ & & $\$ 0$ & & & & \\
\hline Medical outpatient visits & 6.9 & {$[7.2]$} & 7.2 & {$[7.2]$} & 7.4 & {$[8.2]$} & 0.283 & 0.047 & 0.317 \\
\hline & 4.0 & & 5.0 & & 5.0 & & & & \\
\hline Medical outpatient costll & $\$ 1,112$ & {$[\$ 2,183]$} & $\$ 1,161$ & {$[\$ 3,956]$} & $\$ 1,247$ & {$[\$ 2,424]$} & 0.673 & 0.108 & 0.465 \\
\hline & $\$ 403$ & & $\$ 418$ & & $\$ 431$ & & & & \\
\hline Psychotherapy visits & 2.1 & $(4.8)$ & 1.9 & $(4.3)$ & 2.0 & $(4.6)$ & 0.281 & 0.531 & 0.650 \\
\hline & 0.0 & & 0.0 & & 0.0 & & & & \\
\hline Psychotherapy cost & $\$ 132$ & $(\$ 342)$ & $\$ 122$ & $(\$ 320)$ & $\$ 106$ & $(\$ 702)$ & 0.422 & 0.185 & 0.393 \\
\hline & $\$ 0$ & & $\$ 0$ & & $\$ 0$ & & & & \\
\hline Total medical cost 1 I & $\$ 1,251$ & {$[\$ 2,705]$} & $\$ 1,422$ & {$[\$ 4,567]$} & $\$ 1,581$ & {$[\$ 3,769]$} & 0.209 & 0.006 & 0.290 \\
\hline & $\$ 404$ & & $\$ 421$ & & $\$ 432$ & & & & \\
\hline Total medical and drug cost & $\$ 2,008$ & {$[\$ 3,231]$} & $\$ 2,169$ & {$[\$ 4,828]$} & $\$ 2,540$ & {$[\$ 4,269]$} & 0.280 & $<0.001$ & 0.023 \\
\hline & $\$ 1,062$ & & $\$ 1,080$ & & $\$ 1,271$ & & & & \\
\hline
\end{tabular}

* T tests were used to test for equality of means, and chi-squared tests were used to test for equality of proportions.

$\dagger$ Duration of index ADHD drug therapy was defined as time on index therapy until a minimum 60-day gap in the index therapy supply. We report the duration of therapy truncated at 180 days. The overall median duration of approximately 90 days guided our decision to compare 6-month costs.

¥ The 3 most common primary or secondary reasons for inpatient services were major depressive disorder (ICD-9-CM codes 296.2x and 296.3x), ADHD (ICD-9-CM code 314.0x), and depressive disorder (ICD-9-CM code 311.xx).

$\S$ The minimum and maximum values for inpatient services costs for OROS-MPH were $\$ 0$ to $\$ 32,930, \$ 0$ to $\$ 41,804$ for MAS-XR, and $\$ 0$ to $\$ 54,549$ for atomoxetine.

II The minimum and maximum values for medical outpatient/other cost were $\$ 0$ to $\$ 31,756$ for OROS-MPH, $\$ 0$ to $\$ 134,712$ for MAS-XR, and $\$ 0$ to $\$ 36,075$ for atomoxetine.

II The minimum and maximum values for medical cost (inpatient and outpatient/other) were $\$ 0$ to $\$ 35,597$ for OROS-MPH, $\$ 0$ to $\$ 134,712$ for MAS-XR, and $\$ 0$ to $\$ 55$, 950

for atomoxetine. By the criterion of medical cost in excess of $\$ 50,000,2$ patients were medical cost outliers. The diagnosis codes associated with the highest cost claim for the MAS-XR outlier were chronic kidney disease (ICD-9-CM code 585.xx) and iron deficiency anemia (ICD-9-CM code 280.9). The diagnosis codes associated with the highest cost claim for the atomoxetine outlier were acute myocardial infarction (ICD-9-CM code 410.21) and paroxysmal ventricular tachycardia (ICD-9-CM code 427.1).

$A D H D=$ attention-deficit/hyperactivity disorder; ICD-9 = International Classification of Diseases, Ninth Revision, Clinical Modification; MAS-XR = mixed amphetamine salts extended release; OROS-MPH = extended-release methylphenidate.

patients treated with MAS-XR (3.4\%) and atomoxetine (3.3\%, $P=0.031$ ). Atomoxetine- and OROS-MPH-treated patients had higher Charlson Comorbidity Indexes than MAS-XR patients $(P=0.005$ for comparison between atomoxetine and MAS-XR,
$P=0.014$ for comparison between OROS-MPH and MAS-XR). No significant differences were apparent across the 3 groups in the rate of depression or rate of hospital inpatient use in the 6-months before therapy initiation (Table 1). 


\section{Resource Use: 6-Month Postperiod}

The mean (SD) duration of index ADHD drug therapy within 180 days was lower for atomoxetine (96 days [65]) compared with OROS-MPH (107 [66], P<0.001) or MAS-XR (115 [65], $P<0.001$ ) (Table 2). Median duration of therapy was 99 days for OROS-MPH, 128 days for MAS-XR, and 86 days for atomoxetine. Over the 6-month follow-up period, the proportion of patients with at least 1 inpatient place of service claim was not significantly lower in patients treated with OROS-MPH (3.4\%) compared with MAS-XR $(4.8 \%, P=0.058)$, but was lower than the $5.1 \%$ rate for atomoxetine-treated patients $(P=0.024$ for comparison with OROS-MPH). The proportions of patients having an outpatient claim or claim with an emergency room place of service were not significantly different between OROSMPH-treated adults and those treated with MAS-XR or atomoxetine (data not shown).

\section{Descriptive Cost Analysis}

Six-month mean ([SD], median) total health care (medical and drug) costs were $\$ 2,008$ ( $[\$ 3,231], \$ 1,062)$ for OROSMPH-, $\$ 2,169$ ([\$4,828], \$1,080) for MAS-XR-, and $\$ 2,540$ ([\$4,269], \$1,271) for atomoxetine-treated adults (Table 2). Total health care costs were significantly higher for atomoxetinetreated adults compared with those treated with OROS-MPH $(P<0.001)$ and MAS-XR $(P=0.023)$.

The largest component of 6-month total health care costs was attributable to outpatient costs (52\%). No significant differences were observed in outpatient costs or the subset of medical costs attributable to psychotherapy. Drug costs accounted for approximately 38\% of total unadjusted costs for OROS-MPH and atomoxetine versus 34\% for MAS-XR. Mean ([SD], median) ADHD drug costs were higher with atomoxetine at \$392 ([\$298], \$325) compared with MAS-XR at \$322 ([\$250], $\$ 275)$ and OROS-MPH at $\$ 282([\$ 215], \$ 246)(P<0.001$ for both comparisons).

Inpatient hospital cost accounted for $7 \%$ of total direct medical cost for OROS-MPH, 12\% for MAS-XR, and 13\% for atomoxetine. Mean (SD) inpatient service costs were not significantly lower for OROS-MPH patients at $\$ 139(\$ 1,336)$ compared with MAS-XR patients at $\$ 261(\$ 2,060, P=0.053)$, but were lower compared with atomoxetine patients at $\$ 334(\$ 2,480$, $P=0.007)$. The 3 most common reasons for inpatient service use were major depressive disorder (MDD; ICD-9-CM codes 296.2x and 296.3x), ADHD (ICD-9-CM code 314.0x), and depressive disorder (ICD-9-CM code 311.xx).

By using the criteria of medical cost in excess of $\$ 50,000$ or approximately the 99.96 th percentile, 2 patients were medical cost outliers. One patient treated with MAS-XR had total medical and pharmacy costs of $\$ 136,300$, of which $\$ 134,712$ were medical costs; the highest cost claim for this patient was associated with diagnosis codes for chronic kidney disease (ICD-9-CM code 585.xx) and iron deficiency anemia (ICD-9-
CM code 280.9x). One patient who received atomoxetine had total medical and pharmacy costs of $\$ 58,554$, of which $\$ 55,950$ were medical costs; the highest cost claim was associated with diagnosis codes for acute myocardial infarction (ICD-9-CM code 410.21) and paroxysmal ventricular tachycardia (ICD-9CM code 427.1).

\section{Multivariate Regression}

The GLM comparison of health care costs in the 6 months after therapy initiation (adjusting for potential confounders, Table 3) found that risk-adjusted direct health care costs of OROS-MPH-treated adults were on average $\$ 156$ (8.0\%) less than those of MAS-XR-treated adults $(P=0.017)$ and $\$ 226$ (11.3\%) less than those of atomoxetine-treated adults $(P=0.001)$. The health care cost difference was primarily due to differences in medical costs; average medical costs for OROS-MPH-treated adults were $\$ 142$ (10.4\%) less than those of MAS-XR-treated adults $(P=0.022)$ and $\$ 132(9.8 \%)$ less than those of atomoxetine-treated adults $(P=0.033)$.

\section{Discussion}

Our analysis of a privately insured claims database indicates that over the 6-month period following therapy initiation, adults treated with OROS-MPH had, on average, lower all-cause medical and total health care costs than those treated with MAS-XR or atomoxetine after adjusting for patient characteristics. To our knowledge, this is the first study comparing all-cause health care costs of adults diagnosed with ADHD and receiving alternative ADHD drug therapies. ${ }^{28}$ Previous studies have indicated that MPH is a cost-effective treatment for children with ADHD. ${ }^{29-31}$ A study by Marchetti et al. developed a decision-analytic model to estimate the total expected costs for the treatment and management of school-age children with ADHD using 6 commonly prescribed pharmacotherapies: methylphenidate immediate release/extended release (MPH-IR/ER), methylphenidate immediate release (MPH-IR), branded MPH-IR/ER (Metadate CD), OROS-MPH (Concerta), branded MPH-IR (Ritalin), and a combination of dextroamphetamine and amphetamine salts (Adderal). ${ }^{32}$ This study found that dextroamphetamine and amphetamine salts had the highest total expected costs among the ADHD pharmacotherapies evaluated: the average total annual expected cost in 2001 U.S. dollars per treated patient was $\$ 1,710$ for Metadate CD, $\$ 1,876$ for Concerta, $\$ 2,061$ for MPH- IR/ER, \$2,122 for MPH-IR, \$2,392 for Ritalin, and \$2,567 for Adderall.

Other published studies have focused on the direct health care costs of adults with ADHD but did not provide cost comparisons of adults with ADHD on different drug therapies. ${ }^{9-11}$ Those studies compared the costs of care for adults with ADHD with the costs in matched controls and concluded that ADHD is associated with significant economic burden. Compared with the control group, adults diagnosed with ADHD were signifi- 
Health Care Costs of Adults Treated for

Attention-Deficit/Hyperactivity Disorder Who Received Alternative Drug Therapies

\section{TABLE 3 Risk-Adjusted 6-Month Medical and Direct Costs for Adults Diagnosed With ADHD Receiving Different Therapies-Generalized Linear Model}

\begin{tabular}{|c|c|c|c|c|c|}
\hline Medical Costs $\dagger$ & $\begin{array}{c}\text { Risk-Adjusted } \\
\text { Cost Estimate }(\$)^{*}\end{array}$ & \multicolumn{2}{|c|}{ 95\% Confidence Limits (\$) } & $\begin{array}{c}P \text { Value for Comparison } \\
\text { With OROS-MPH }\end{array}$ & $\begin{array}{c}P \text { Value for Comparison } \\
\text { With MAS-XR }\end{array}$ \\
\hline OROS-MPH & 1,220 & 1,141 & 1,305 & & \\
\hline MAS-XR & 1,362 & 1,276 & 1,453 & 0.022 & \\
\hline Atomoxetine & 1,352 & 1,267 & 1,443 & 0.033 & 0.880 \\
\hline \multicolumn{6}{|c|}{ Medical + Drug Costs } \\
\hline OROS-MPH & 1,782 & 1,696 & 1,872 & & \\
\hline MAS-XR & 1,938 & 1,848 & 2,033 & 0.017 & \\
\hline Atomoxetine & 2,008 & 1,915 & 2,106 & 0.001 & 0.307 \\
\hline
\end{tabular}

* The risk-adjusted costs were estimated by a GLM with a log link and gamma distribution adjusting for patient demographic characteristics (i.e., age, gender, region) and comorbidities (substance abuse, depression, and Charlson Comorbidity Index).

$\dagger$ Medical costs include inpatient, outpatient and other costs.

\# Total direct costs include medical costs and drug costs.

$\S$ A sensitivity analysis excluding the 2 patients with medical costs in excess of \$50,000 suggested that of the \$156 6-month cost difference between OROS-MPH and MAS-XR and the \$226 6-month cost difference between OROS-MPH and atomoxetine, $\$ 47$ (30\%) and \$11 (5\%), respectively, were attributable to high-cost outliers.

$A D H D=$ attention-deficit/hyperactivity disorder; GLM=generalized linear model; MAS-XR=mixed amphetamine salts extended release; OROS-MPH=extendedrelease methylphenidate.

cantly more likely to have a comorbid diagnosis of asthma, anxiety, bipolar disorder, depression, drug or alcohol abuse, antisocial disorder, or oppositional disorder (e.g., $4.7 \%$ vs. $2.9 \%$ for asthma and $4.5 \%$ vs. $0.58 \%$ for bipolar disorder). ${ }^{9}$ Adjusting for patient characteristics, adults with ADHD had an excess medical cost of $\$ 2,880(P<0.001) .{ }^{9}$ Costs associated with accident claims are more than 3 times higher in adults with ADHD than in controls. ${ }^{10}$ The total excess cost of ADHD in the United States in 2000 was $\$ 31.6$ billion, including the higher costs associated with family members of persons with ADHD. ${ }^{11} \mathrm{~A}$ recently published paper also reported national estimates and characteristics of ambulatory visits by adults with ADHD in the United States but did not compare the cost of alternative ADHD drug treatments. ${ }^{33}$ Our study assessed the real-world drug and total medical costs associated with initiation of 3 alternative drug therapies for adult patients with a diagnosis of ADHD.

\section{Limitations}

Foremost among the limitations of our study was its short duration. The overall median length of index ADHD drug therapy was approximately 90 days, ranging from a median of 86 days for atomoxetine to 99 days for OROS-MPH to 128 days for MAS-XR. We anticipated this relatively short therapy period based on a previous study by Perwien et al., which concluded that even though ADHD patients continued their ADHD medication for several months, they did not consistently take medication for more than 2 months. ${ }^{24}$

Second, our study did not directly assess the clinical severity of ADHD. Like all administrative claims data- bases, ADHD severity could not be determined from the data that we accessed. To assess and control for subjects' physical conditions, we used a common proxy of comorbidity risk measurement, the Charlson Comorbidity Index, and selected comorbidities. ${ }^{5,9,11}$ However, other risk-adjustment alternatives to the Charlson Comorbidity Index also exist. ${ }^{34,35}$ Studies have found similar mortality predictive accuracy with the RomanoCharlson Comorbidity Index (used in our analysis) and the Elixhauser comorbidities. ${ }^{36,37}$

Third, we did not restrict ICD-9-CM codes specifically to ADHD. We included ICD-9-CM codes 314.00 (attentiondeficit disorder without hyperactivity, also known as ADD of predominantly the inattentive type ${ }^{38}$ ) and 314.0 (nonspecific attention-deficit disorder) as well as 314.01, which is specific to attention-deficit disorder with hyperactivity (ADHD). While other researchers have used ICD-9-CM codes 314.0x to describe ADHD, ${ }^{24,25}$ the correct definition of code 314.0x is ADD because ICD-9-CM code 314.00 is intended to specify ADD without hyperactivity. As is true of much of the research in this area, we did not analyze the data with respect to the use of ICD-9-CM code 314.00 versus 314.01.

Fourth, we did not require health care or hospital costs to be related to ADD/ADHD; hence, we report all-cause health care and hospital costs. While ADD/ADHD (ICD-9-CM code $314.0 \mathrm{x}$ ) was 1 of the 3 most common primary or secondary reasons for hospital inpatient service use, we did not exclude any hospital service use during the follow-up period. The other 2 most common principal reasons for inpatient service use were MDD (ICD-9-CM codes 296.2x and 296.3x) and depres- 
sive disorder (ICD-9-CM code 311.xx). A significantly higher proportion of atomoxetine patients had inpatient services during the follow-up period, and the average inpatient service costs for OROS-MPH were lower compared with MAS-XR and atomoxetine. Calculating the cost of only claims with an ADHD diagnosis, psychotherapy visits, and ADHD medications is likely to underestimate ADHD-related costs considering that research has shown that ADHD is associated with an increased risk of accidents. ${ }^{10}$ Another study has demonstrated that only a small percentage of total health care costs were clearly disorder specific. The authors concluded that either calculating the costs of medical services used in disease management underestimates the full economic impact of the disorder or that the relationship between the disorder and total health care cost was not causal. ${ }^{39}$ We did not use this method of aggregating and reporting disease-specific versus other-cause utilization and costs, which may have helped to better inform the discussion about ADD/ADHD-related costs and costs that are not directly attributable to ADD/ADHD.

Fifth, the inclusion of 2 patients with outlier medical costs, defined as $\$ 50,000$ or more per patient, was partly responsible for the differences in mean costs observed in the descriptive analysis. For example, 1 patient in the MAS-XR group had medical costs of $\$ 134,712$ and total medical and pharmacy costs of $\$ 136,300$, which was approximately $\$ 95,924$ more than the highest-cost outlier for OROS-MPH (\$35,597 in medical costs, $\$ 40,776$ in total medical and pharmacy costs) or approximately $\$ 77,746$ more thanthe atomoxetine patient (\$55,950 in medical costs, $\$ 58,554$ in total medical and pharmacy costs (see Table 2). Differences in median 6-month total health care costs, which are not as sensitive as the means to outliers, ranged from $\$ 12$ to $\$ 209$. However, our multivariate analysis adjusted for the skewness that is typical of cost data, controlling for demographic characteristics, substance abuse, depression, and the Charlson Comorbidity Index.

Sixth, the categorization of inpatient services and outpatient/ other services relied on place-of-service codes. To the extent that some emergency room and outpatient visits were coded with a place of service designated as hospital inpatient, rehabilitation center, residential treatment center, or psychiatric facility, their costs were categorized into inpatient services costs. However, inpatient service and outpatient/other service costs were mutually exclusive categories; therefore, our conclusions based on medical and total health care costs remain valid.

Seventh, potential selection bias cannot be fully adjusted. Even though we adjusted for patient baseline characteristics, Charlson comorbidity index, and selected comorbidities, ${ }^{5,9,11}$ those adjustments were based on diagnoses provided in the 6 months prior to therapy initiation. Therefore, potential baseline differences among the 3 groups may not be fully adjusted due to the limited information available.

Eighth, care must be taken in generalizing the results of this study because the sample is not representative of the entire U.S. population. Our data did not include low-income, Medicaid, and Medicare populations. In addition, the cost (i.e., provider payment) measurement did not include patient deductible or coinsurance amounts, nor did it reflect provider-submitted charges.

Ninth, we do not know the formulary status of these drugs. If one drug was more likely to be a nonformulary drug with a higher copayment, the use of net payer cost after subtraction of member cost share could introduce a systematic bias that would show lower plan cost for the nonformulary drug.

Last, it is possible that mental illness in general and ADHD specifically may be underreported in claims data due to social stigma, practice differences between primary care physicians and specialists, and other factors. The purpose of our study is to compare the costs associated with alternative ADHD drug therapy for patients receiving a diagnosis of ADHD. There appears to be no reason to believe that this potential problem concerning the underreporting of ADHD in medical claims would be distributed disproportionately among our 3 drug treatment groups. Further studies might be conducted to assess the cost implications of the possible under-reporting of ADHD.

\section{Conclusions}

Over the 6-month period following drug therapy initiation, adults with a diagnosis of ADHD initiated on OROS-MPH had, on average, $8 \%$ lower total all-cause drug and medical costs compared with MAS-XR or $11 \%$ lower total costs compared with atomoxetine. Further research is needed to evaluate clinical and economic outcomes in adults diagnosed with ADHD who are receiving alternative therapies.

\section{Authors}

ERIC Q. WU, PhD, is a vice president; HOWARD G. BIRNBAUM, $\mathrm{PhD}$, is a vice president; JASMINA I. IVANOVA, MA, is an associate; and ELAINE YANG, PhD, is an associate, Analysis Group, Inc., Boston, Massachusetts. HUABIN F. ZHANG, MD, MPH, is a director, World Wide Health Economics \& Pricing, Virology, Johnson \& Johnson Pharmaceutical Services, LLC, Raritan, New Jersey; DAVID MALLET, $M B A$, is a managing director, Employer Solutions Division, Ingenix, Inc., Rocky Hill, Connecticut.

AUTHOR CORRESPONDENCE: Eric Q. Wu, PhD, Vice President, Analysis Group, Inc., 111 Huntington Ave., 10th Fl., Boston, MA 02199. Tel: (617) 425-8000; Fax: (617) 425-8001;

E-mail: ewu@analysisgroup.com

\section{DISCLOSURES}

Funding for this research was provided by McNeil Pediatrics Division of McNeil-PPC, Inc. to Analysis Group, Inc. Huabin Zhang is a former employee of McNeil-PPC. Authors Wu, Birnbaum, Ivanova, and Yang are employees of Analysis Group, Inc.

Author Eric Wu served as principal author of the study. Study concept and design were contributed primarily by $\mathrm{Wu}$ and authors Howard Birnbaum, 
Huabin Zhang, and David Mallet. Data collection and analysis was the work of authors Elaine Yang, Jasmina Ivanova, and Mallet; data interpretation was primarily the work of $\mathrm{Wu}$, with input from Birnbaum, and Ivanova. Writing of the manuscript and its revision was primarily the work of Ivanova, with input from $\mathrm{Wu}$, Birnbaum, Zhang, and Yang.

\section{REFERENCES}

1. Kessler RC, Adler L, Ames M, et al. The prevalence and effects of adult attention deficit/hyperactivity disorder on work performance in a nationally representative sample of workers. J Occup Environ Med. 2005;47(6):565-72.

2. Doyle R. The history of adult attention-deficit/hyperactivity disorder. Psychiatr Clin North Am. 2004;27(2):203-14.

3. McGough JJ, Barkley RA. Diagnostic controversies in adult attention deficit hyperactivity disorder. Am J Psychiatry. 2004;161:1948-56.

4. Adler L, Cohen J. Diagnosis and evaluation of adults with attention-deficit/ hyperactivity disorder. Psychiatr Clin North Am. 2004;27(2):187-201.

5. Biederman J. Impact of comorbidity in adults with attention-deficit/ hyperactivity disorder. J Clin Psychiatry. 2004;65(S3):3-7.

6. Weiss MD, Weiss JR. A guide to the treatment of adults with ADHD. J Clin Psychiatry. 2004;65(S3):27-37.

7. Wilens TE, Dodson W. A clinical perspective of attention-deficit/hyperactivity disorder into adulthood. J Clin Psychiatry. 2004;65(10):1301-13.

8. Spencer TJ, Biederman J, Wilens T. Stimulant treatment of adult attentiondeficit/hyperactivity disorder. Psychiatr Clin North Am. 2004;27(2):361-72.

9. Secnik K, Swensen A, Lage MJ. Comorbidities and costs of adult patients diagnosed with attention-deficit hyperactivity disorder. Pharmacoeconomics. 2005:23(1):93-102

10. Swensen A, Birnbaum HG, Ben Hamadi R, Greenberg P, Cremieux P-Y, Secnik K. Incidence and costs of accidents among attention-deficit/hyperactivity disorder patients. J Adolesc Health. 2004;35(2):346el-e9.

11. Birnbaum HG, Kessler RC, Lowe SW, et al. Costs of attention deficithyperactivity disorder (ADHD) in the US: excess costs of persons with ADHD and their family members in 2000. Curr Med Res Opin. 2005;21(2):195-206.

12. Swensen A, Birnbaum HG, Secnik K, et al. Attention-deficit/hyperactivity disorder (ADHD): increased costs for patients and their families. J Am Acad Child Adolesc Psychiatry. 2003;42(12):1415-23.

13. Wilens TE, Spencer TJ, Biederman J. A review of the pharmacotherapy of adults with attention-deficit/hyperactivity disorder. J Atten Disord. 2002; 5:189-202

14. Safren SA, Sprich S, Chulvic S, Otto MW. Psychosocial treatments for adults with attention-deficit/hyperactivity disorder. Psychiatr Clin North Am. 2004;27(2):349-60.

15. Spencer TJ. ADHD treatment across the life cycle. J Clin Psychiatry. 2004;65(suppl 3):22-26

16. Wilens T, McBurnett K, Stein M, Lerner M, Spencer T, Wolraich M. ADHD treatment with once-daily OROS methylphenidate: final results from a longterm open-label study. J Am Acad Child Adolesc Psychiatry. 2005;44(10):1015-23.

17. Christman AK, Fermo JD, Markowitz JS. Atomoxetine, a novel treatment for attention-deficit/hyperactivity disorder. Pharmacotherapy. 2004;24(8):1020-36.

18. Stein MA. Innovations in attention-deficit/hyperactivity disorder pharmacotherapy: long-acting stimulant and nonstimulant treatments. Am J Manag Care. 2004:10:S89-S98

19. Greenhill LL, Pliszka S, Dulcan MK, et al. Practice parameter for the use of stimulant medications in the treatment of children, adolescents, and adults. J Am Acad Child Adolesc Psychiatry. 2002;41(2 suppl):26S-49S.

20. Little R, Rubin D. Statistical Analysis with Missing Data. New York: John Wiley \& Sons; 1987:62-67.
21. Wedderburn RWM. Quasi-likelihood functions, generalized linear models, and the Gauss-Newton method. Biometrika. 1974;61:439-47.

22. Manning WG, Mullahy J. Estimating log models: to transform or not to transform? J Health Econ. 2001;20:461-94.

23. Manning WG. The logged dependent variable, heteroscedasticity, and the retransformation problem. J Health Econ. 1998;17:283-95.

24. Perwien A, Hall J, Swensen A, Swindle R. Stimulant treatment patterns and compliance in children and adults with newly treated attention-deficit/ hyperactivity disorder. J Manag Care Pharm. 2004;10(2):122-29. Available at: http://www.amcp.org/data/jmcp/Research-122-129.pdf.

25. Van Brunt DL, Johnston JA, Ye W, Pohl GM, O'Hara NN. Factors associated with initiation with atomoxetine versus stimulants in the treatment of adults with ADHD: retrospective analysis of administrative claims data. J Manag Care Pharm. 2006;12(3):230-38. Available at: http://www.amcp.org/data/jmcp/ Research_230-238.pdf.

26. Charlson ME, Pompei P, Ales KL, McKenzie CR. A new method of classifying prognostic comorbidity in longitudinal studies: development and validation. J Chron Dis. 1987;40(5):373-83.

27. Romano PS, Roos LL, Jollis J. Adapting a clinical comorbidity index for use with ICD-9-CM administrative data. J Clin Epidemiol. 1993;46(10):1075-79.

28. Matza LS, Paramore C, Prasad M. A review of the economic burden of ADHD. Cost Eff Resour Alloc. 2005;9:3-5.

29. Gilmore A, Milne R. Methylphenidate in children with hyperactivity: review and cost-utility analysis. Pharmacoepidemiol Drug Saf. 2001;10:85-94

30. Lord J, Paisley S. The Clinical Effectiveness and Cost-Effectiveness of Methylphenidate for Hyperactivity in Childhood, Version 2. London, England: National Institute for Clinical Excellence; 2000.

31. Zupancic JAF, Miller A, Raina P, Lee SK, Klassen A, Olsen L. Part 3: Economic evaluation of pharmaceutical and psychological/behavioural therapies for attention-deficit/hyperactivity disorder. In: Miller A, Lee SK, Raina $\mathrm{P}$, et al., eds. A Review of Therapies for Attention-Deficit/Hyperactivity Disorder. Ottawa, Canada: Canadian Coordinating Office for Health Technology Assessment; 1998

32. Marchetti A, Magar R, Lau H, et al. Pharmacotherapies for attention-deficit/ hyperactivity disorder: expected-cost analysis. Clin Ther. 2001;23(11):1904-21

33. Sankaranarayanan J, Puumala SE, Kratochvil CJ. Diagnosis and treatment of adult attention-deficit/hyperactivity disorder at US ambulatory care visits from 1996 to 2003. Curr Med Res Opin. 2006;22(8):1475-91.

34. Elixhauser A, Steiner C, Harris DR, Coffey RM. Comorbidity measures for use with administrative data. Med Care. 1998;36:8-27.

35. Averill RF, Goldfield N, Hughes JS, et al. All patient refined diagnosis related groups (APR-DRGs). Version 20.0. Methodology overview. Available at: http://www.hcup-us.ahrq.gov/db/nation/nis/APR-DRGsV20MethodologyOverviewandBibliography.pdf. Accessed April 6, 2007.

36. Schneeweiss S, Wang PS, Avorn J, Glynn RJ. Improved comorbidity adjustment for predicting mortality in Medicare populations. Health Serv Res. 2003;38(4):1103-20.

37. Yan Y, Birman-Deych E, Radford MJ, Nilasena DS, Gage BF. Comorbidity indices to predict mortality from Medicare data: results from the national registry of atrial fibrillation. Med Care. 2005;43(11):1073-77.

38. ICD9data.com - free ICD-9-CM database. Available at: http://www. icd9data.com/2007/Volume1/290-319/300-316/314/default.htm. Accessed June 27, 2007.

39. Nissenson AR, Wade S, Goodnough T, Knight K, Dubois RW. Economic burden of anemia in an insured population. J Manag Care Pharm. 2005; 11(7):565-74. Available at: http://www.amcp.org/data/jmcp/Original\%20Research_ 565_574.pdf. 